Supporting Information

\title{
A Fluorescent Probe Based on a Water-Soluble Conjugated Polymer: from Construction to Ultra-low Concentration
}

\section{Biomarker Non-invasive Detection}

Mi Zhou ${ }^{\text {a,* }}$, Zejian $\mathrm{He}^{\text {a }}$, Ping Chen ${ }^{\mathrm{b}}$, $\mathrm{Na} \mathrm{Wu}{ }^{\text {a }}$, Liangliang Zhu ${ }^{\text {a }}$, Yifan Zhang a ${ }^{\text {, Jie }} \mathrm{Li}^{\text {a }}$

${ }^{a}$ College of Materials Science and Engineering, Zhejiang University of Technology, Zhejiang 310014, P. R. China.

b Department of Clinical Laboratory, Zhejiang University of Traditional Chinese Medicine First Affiliated Hospital, Zhejiang 310014, P. R. China.

* Corresponding author. E-mail: zhoumi@zjut.edu.cn (M. Zhou)

\section{EXPERIMENTAL}

\subsection{Materials}

Experimental 7-bromo-9,9-dihexylfluoren-2-ylboronic acid pinacol ester was prepared in the way similar to the literature. ${ }^{39} \mathrm{Pd}_{2}(\mathrm{dba})_{3}, \mathrm{t}-\mathrm{Bu}_{3} \mathrm{P}$, bromobenzyl alcohol, phenylboronic acid, $\mathrm{K}_{3} \mathrm{PO}_{4}$, glucose oxidase (Glu-Ox), uric acid oxidase (UA-Ox), galactose oxidase (Gal-Ox), glucose, uric acid, galactose, hydrogen peroxide $\left(\mathrm{H}_{2} \mathrm{O}_{2}\right)$, methyl iodide $\left(\mathrm{CH}_{3} \mathrm{I}\right)$, 
a-bromoisobutyryl bromide, triethylamine and 1,1,4,7,7-Pentamethyldiethylenetriamine (PMDETA) were purchased from Aldrich and used as received without further purification.

$\mathrm{CuBr}$ (Aldrich, 98\%) was purified by stirring with glacial acetic acid, washing with ethanol, and then drying in a vacuum at $70{ }^{\circ} \mathrm{C}$. 2-(Dimethylamino)ethyl methacrylate (DMAEMA, Aldrich, 99\%), was distilled at reduced pressure to remove inhibitor. THF was distilled from $\mathrm{Na}$ /benzophenone under nitrogen before used, other solvents used without further purification.

\subsection{Apparatus}

Transmission electron microscopy (TEM) image were recorded on a JEM 1010 microscope operating at $80 \mathrm{kV}$. The samples for TEM were prepared by drop casting of solution onto carbon coated copper grids placed on a piece of filter paper to remove excess solvent. ${ }^{1} \mathrm{H}$ NMR spectra were recorded on AVANCE III (Bruker, Switzerland) spectrometer at 500 MHz. Molecular weights and polydispersities were determined using a THF GPC setup operating at $35{ }^{\circ} \mathrm{C}$ and comprising Styragel HR1, HR2, HR4 column, waters 2414 refractive index detector and a waters 1515 pump. The GPC eluent was THF (2 v/v\% triethylamine) at a flow rate of $1.0 \mathrm{~mL} \mathrm{~min}{ }^{-1}$. Calibration was conducted using 11 near-monodisperse poly(styrene) standards $\left(\mathrm{M}_{\mathrm{p}}=580\right.$ to $\left.281000 \mathrm{~g} \mathrm{~mol}^{-1}\right)$, and breeze2 software. UV-visible 
absorption and fluorescene spectra of the copolymer solutions were recorded by a UV-visible spectrophotometer (Jasco V-570) and a fluorescence spectrophotometer (Hitachi F-4600, Japan), respectively.

\subsection{Synthesis of ATRP macroinitiator (PF-Br)}

The synthesis for hydroxy-terminated poly[2,7-(9,9-dihexyl-fuorene)] (PF-OH) was prepared by previously reported. Briefly, in a drybox under Ar atmosphere, to $5 \mathrm{~mL}$ vial containing $\mathrm{Pd}_{2}(\mathrm{dba})_{3}(5.5 \mathrm{mg}, 0.006 \mathrm{mmol})$, THF $(0.4 \mathrm{~mL})$, t-Bu 3 P $(48 \mu \mathrm{L}, 0.5 \mathrm{M}$ solution in THF) was added $p$-bromoiodobenzene $(40 \mu \mathrm{L}, 0.25 \mathrm{M}$ solution in THF). The mixture was stirred for $1 \mathrm{~h}$ at room temperature to generate the initiator. In another vial containing the monomer $(0.1 \mathrm{mmol})$ and THF $(5.4 \mathrm{~mL})$ was added $0.5 \mathrm{~mL}$ of $\mathrm{K}_{3} \mathrm{PO}_{4}$ solution $(0.5 \mathrm{~mL}, 2 \mathrm{M}$ solution in water) and the mixture was cooled to $0{ }^{\circ} \mathrm{C}$. The solution of the in situ generated initiator was quickly injected into the solution of monomer and the resulting mixture was stirred for $30 \mathrm{~min}$ at $0{ }^{\circ} \mathrm{C}$ before quenched by phenylboronic acid. After extracted, washed, and dried, the solid product (PF-OH) was obtained by precipitating in methanol and drying under vacuum. To prepare the ATRP macroinitiator (PF-Br), the PF-OH was modificated by a-bromoisobutyryl bromide. Typically, the PF-OH $(0.5 \mathrm{~g}, 0.1 \mathrm{mmol})$ was dissolved in $20 \mathrm{~mL}$ THF and treated with triethylamine $(101 \mathrm{mg}, 1 \mathrm{mmol})$ and $\alpha$-bromoisobutyryl bromide (207 
$\mathrm{mg}, 0.9 \mathrm{mmol}$ ) before the solution was stirred at $40{ }^{\circ} \mathrm{C}$ under an atmosphere of Ar for $16 \mathrm{~h}$. After filtrated, the mixture solution was concentrated and added drop-wise to methanol with stirring. The precipitate (PF-Br) was collected by filtration, washed with methanol and water and dried under vacuum.

\subsection{Synthesis of PF-b-PDMAEMA block copolymer}

The macroinitiator PF-Br $(0.1 \mathrm{~g}$, almost $0.02 \mathrm{mmol})$ was combined with THF $(5.0 \mathrm{~mL})$,

DMAEMA $(5.5 \mathrm{~mL}, 32.9 \mathrm{mmol})$ and $\mathrm{CuBr}(5.9 \mathrm{mg}, 0.04 \mathrm{mmol})$ in a dry flask. The reaction mixture was subjected to three consecutive freeze-pump-thaw cycles in Ar atmosphere before it was pumped into an inert atmosphere glovebox and charged with PMDETA ( $25 \mu \mathrm{L}, 0.124$ mmol). The mixture was subjected to another freeze-pump-thaw cycle before it was heated to $80{ }^{\circ} \mathrm{C}$ with oil bath. The flask was then stirred in the $80{ }^{\circ} \mathrm{C}$ oil bath for $6 \mathrm{~h}$ before it was rapidly cooled in an ice bath. The mixture solution was dialyzed by water to remove the residual $\mathrm{Cu}$ complexes and monomer and so on. After dialyzed, the mixture solution was turned to white emulsion. The product PF-b-PDMAEMA was collected by freezen-dry with a dryer. 

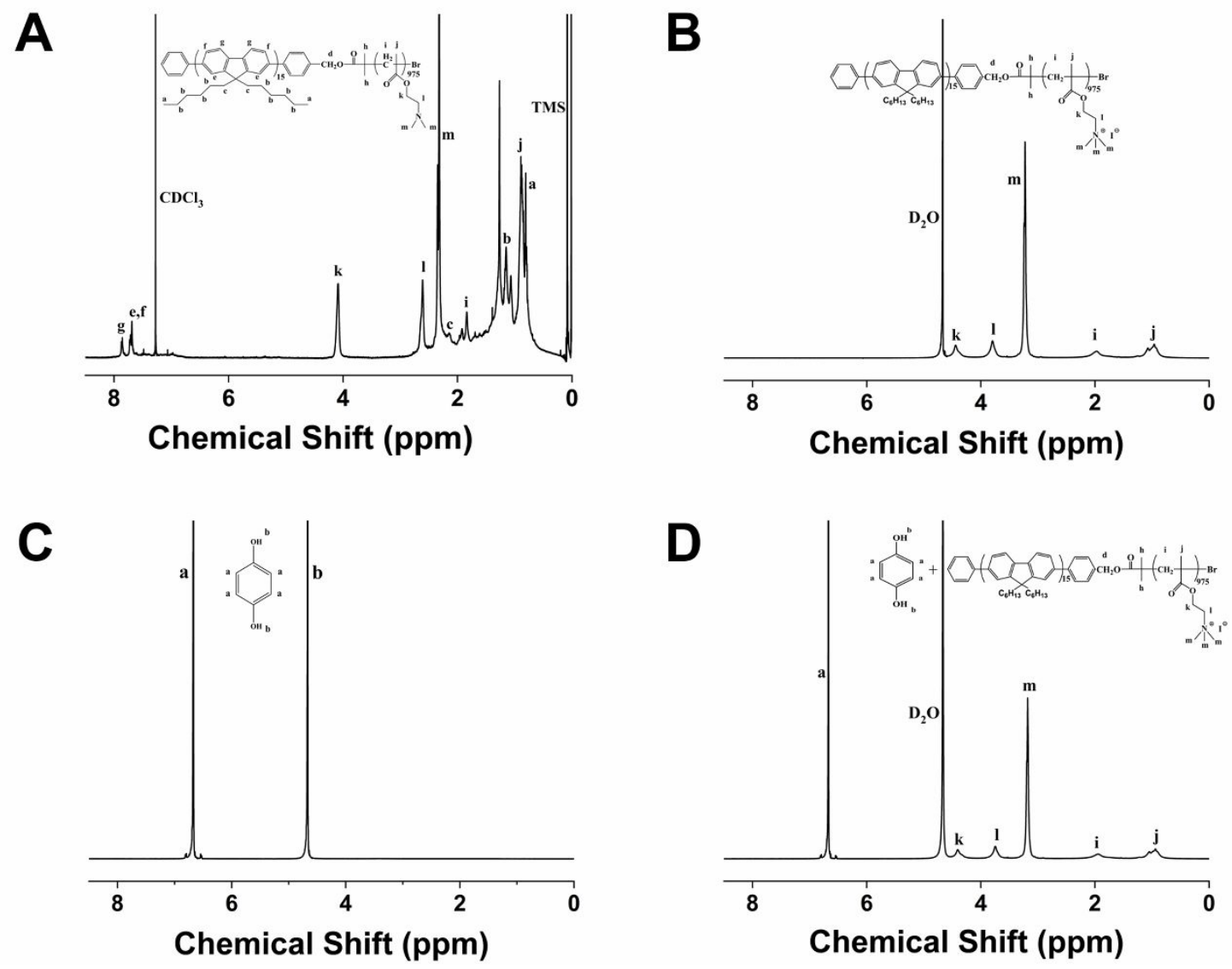

Fig. S1. The ${ }^{1} \mathrm{H}$ NMR spectra of (A) PF- $b$-PDMAEMA, (B) PF- $b$-PDI, (C) hydroquinone, (D) PF- $b$-PDI with hydroquinone.

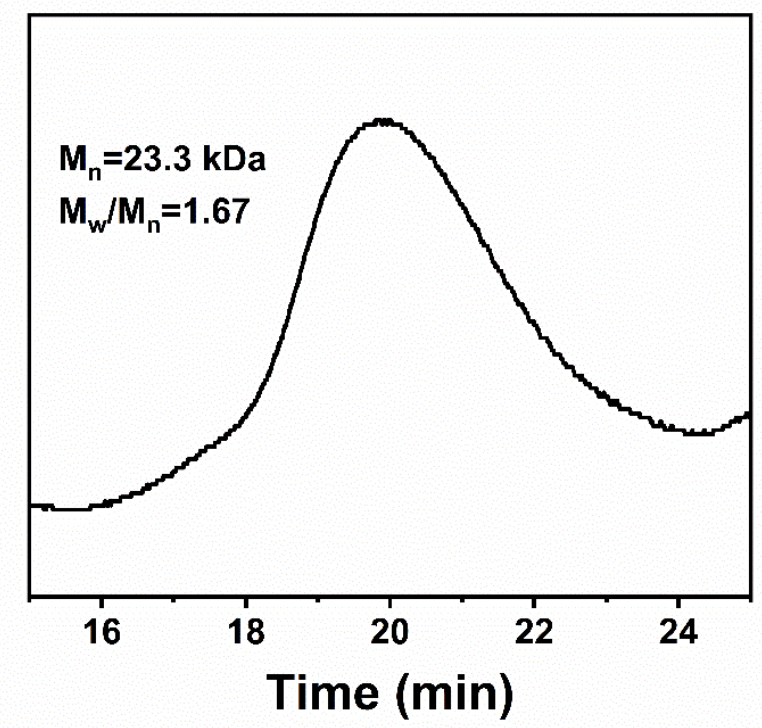

Fig. S2. The GPC trace of PF- $b$-PDMAEMA. 


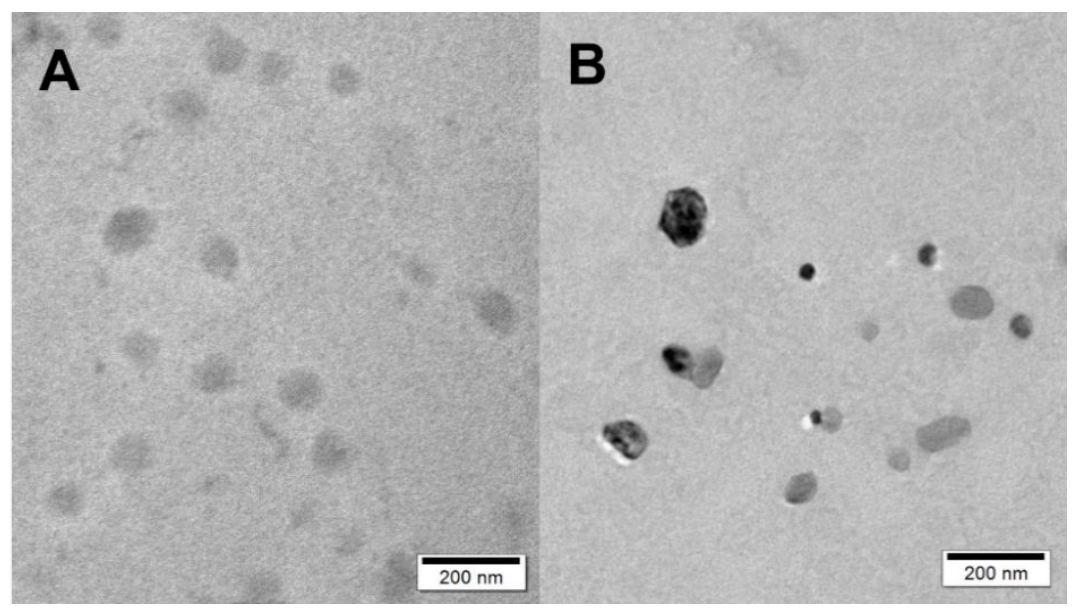

Fig. S3 The TEM image of micelles before (A) and after (B) loading of HQ.
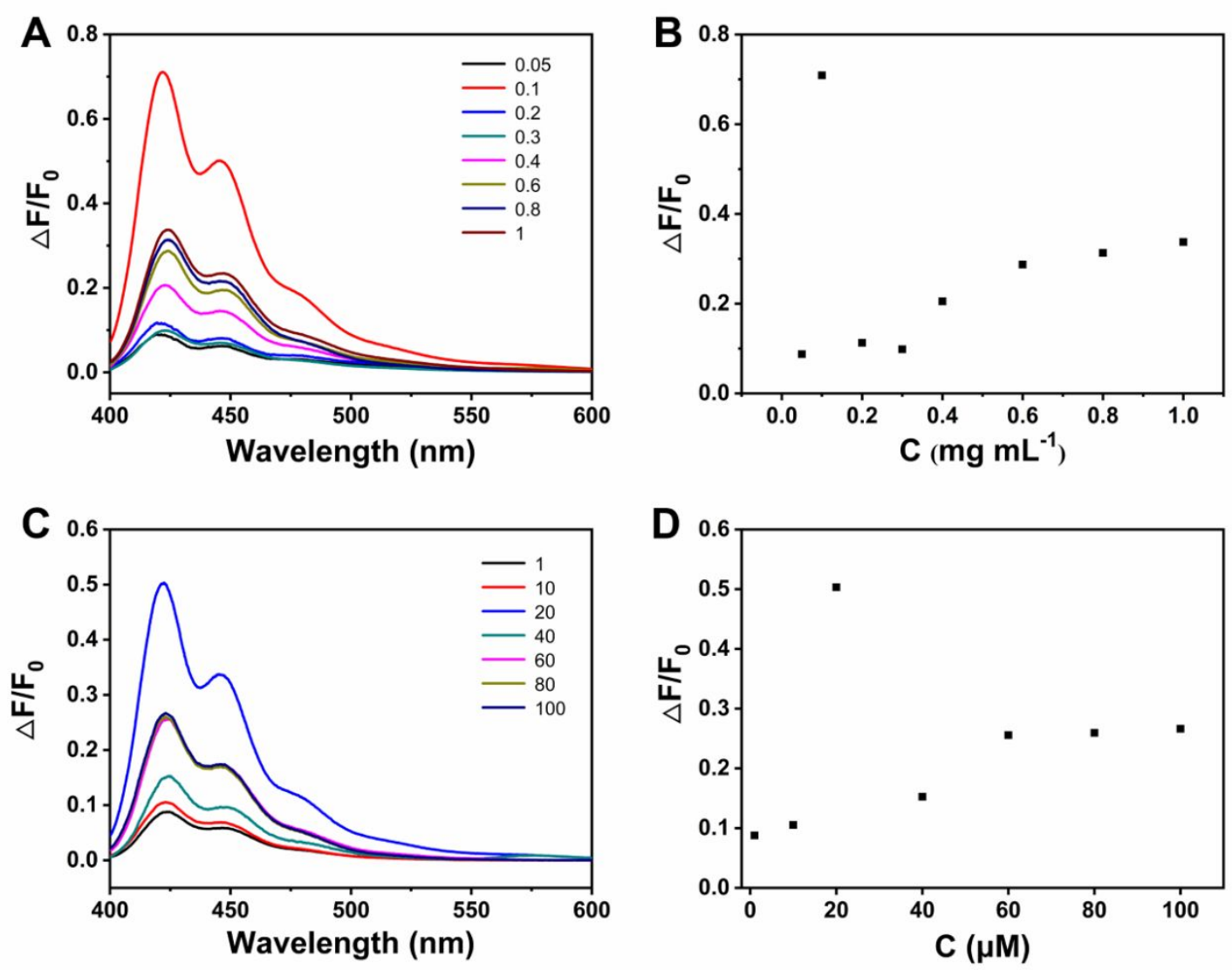

Fig. S4. (A) Relative fluorescence responses of $\mathrm{HQ}$ with $\mathrm{H}_{2} \mathrm{O}_{2}$ after adding different concentrations $\left(0.05-1 \mathrm{mg} \mathrm{mL}^{-1}\right)$ of PF- $b$-PDI; (B) The effect of concentration of PF- $b$-PDI on fluorescence intensity. Experimental conditions: $0.1 \mathrm{mM} \mathrm{HQ}$. (C) Relative fluorescence responses of PF- $b$-PDI with $\mathrm{H}_{2} \mathrm{O}_{2}$ after adding different concentrations (1-100 $\left.\mu \mathrm{M}\right)$ of HQ; (D) The effect of concentration of HQ. Experimental conditions: $0.1 \mathrm{mg} \mathrm{mL}^{-1} \mathrm{PF}-b$-PDI. $\mathrm{F}_{0}$ is the intensity in the absence of $\mathrm{H}_{2} \mathrm{O}_{2}$, and $\mathrm{F}$ is the intensity in the presence of $\mathrm{H}_{2} \mathrm{O}_{2}$. 

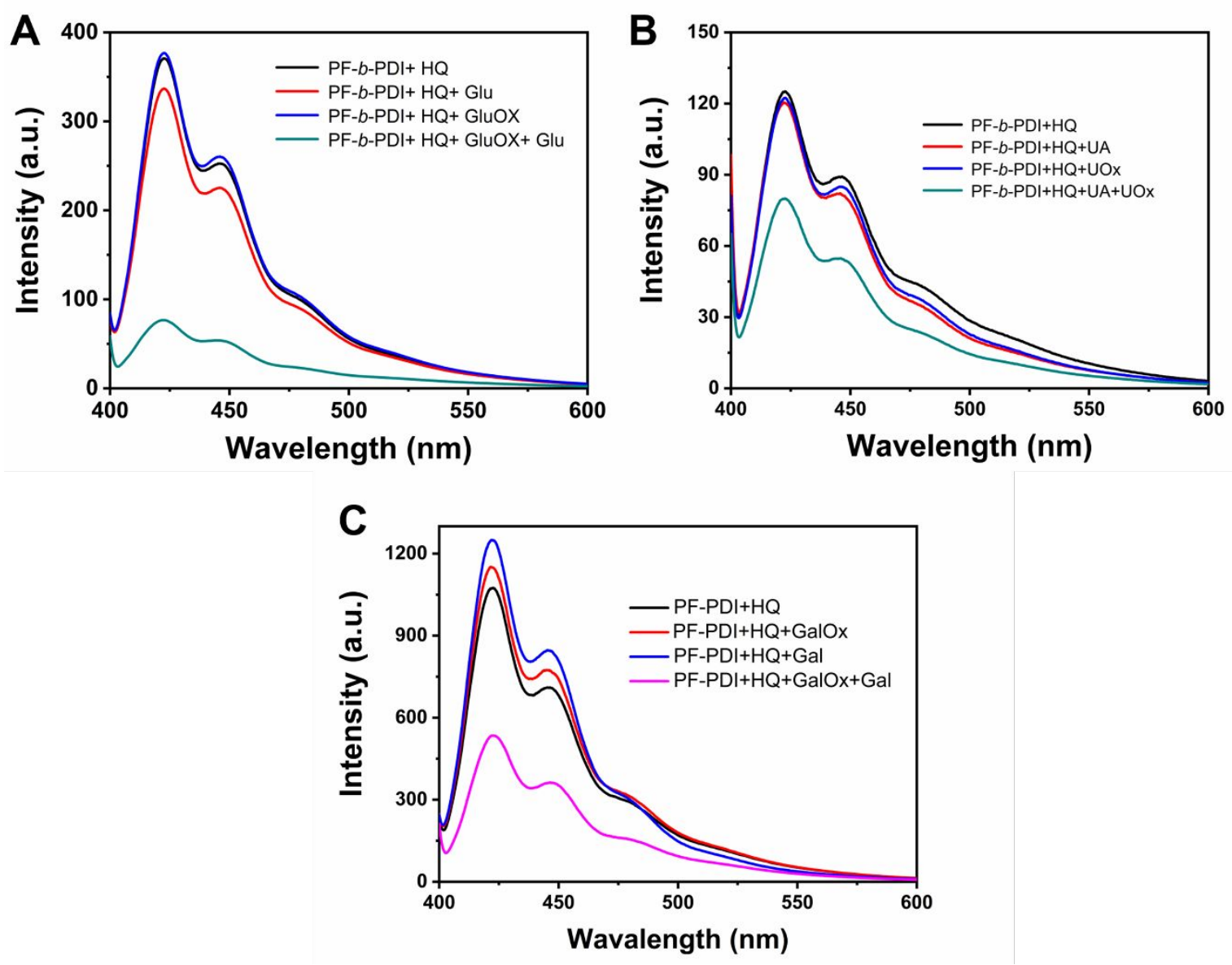

Fig. S5. (A) Fluorescence spectra of PF- $b$-PDI with hydroquinone, PF- $b$-PDI with hydroquinone, glucose, PF- $b$-PDI with hydroquinone, Glu-Ox, PF- $b$-PDI with hydroquinone, glucose, PF- $b$-PDI with hydroquinone, glucose, Glu-Ox; (B) Fluorescence spectra of PF- $b$-PDI with hydroquinone, PF- $b$-PDI with hydroquinone, uric acid, PF- $b$-PDI with hydroquinone, UOx, PF- $b$-PDI with hydroquinone, uric acid, PF- $b$-PDI with hydroquinone, uric acid, UOx; (C) Fluorescence spectra of PF- $b$-PDI with hydroquinone, PF- $b$-PDI with hydroquinone, galactose, PF- $b$-PDI with hydroquinone, Gal-Ox, PF- $b$-PDI with hydroquinone, galactose, PF- $b$-PDI with hydroquinone, galactose, Gal-Ox. 


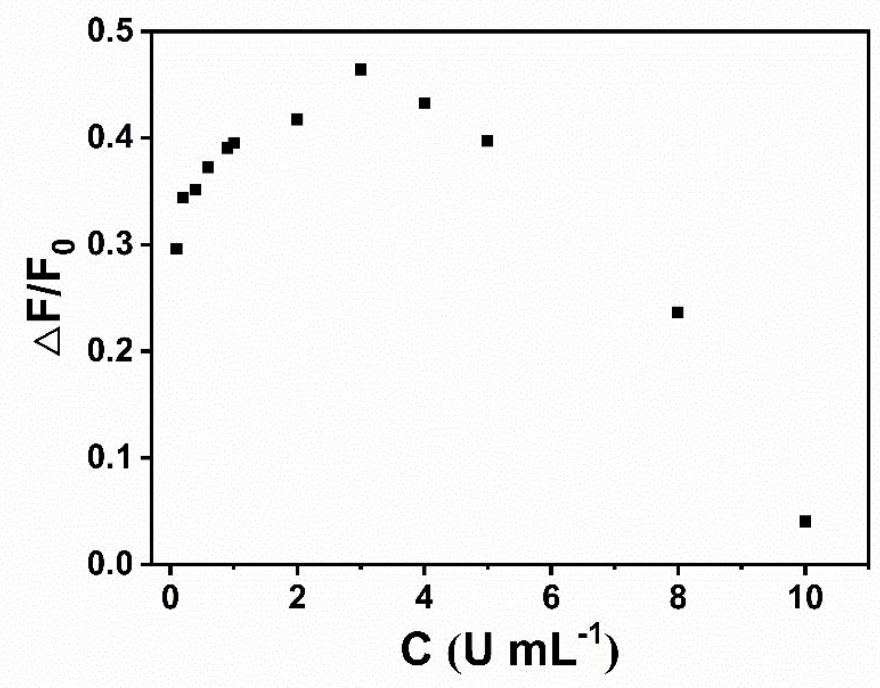

Fig. S6. The effect of concentration of Glu-Ox. Experimental conditions: PF- $b$-PDI, $0.1 \mathrm{mg}$ $\mathrm{mL}^{-1}$; hydroquinone, $20 \mu \mathrm{M}$.

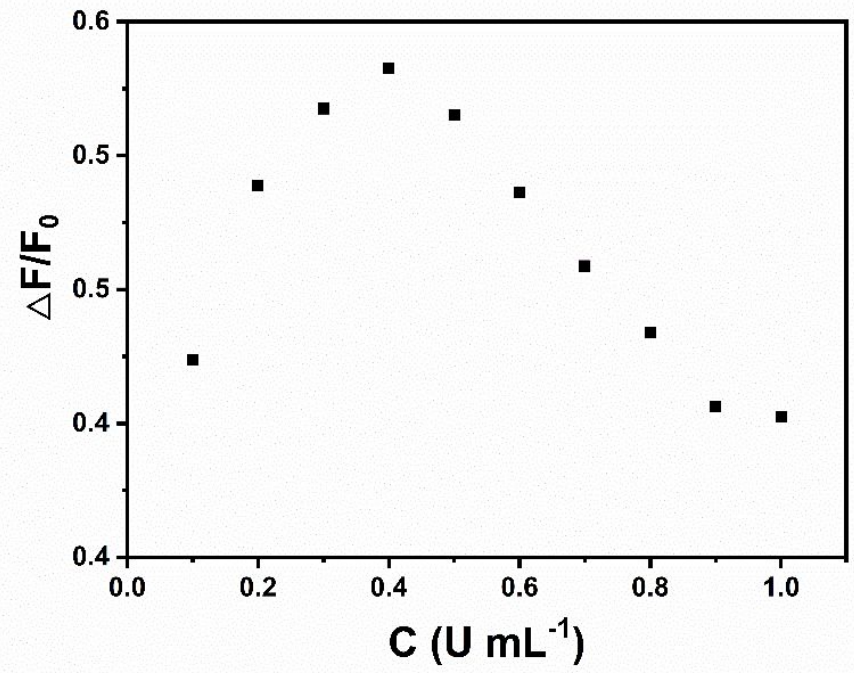

Fig. S7. The effect of concentration of UA-Ox. Experimental conditions: PF- $b$-PDI, $0.1 \mathrm{mg}$ $\mathrm{mL}^{-1}$; hydroquinone, $20 \mu \mathrm{M}$. 


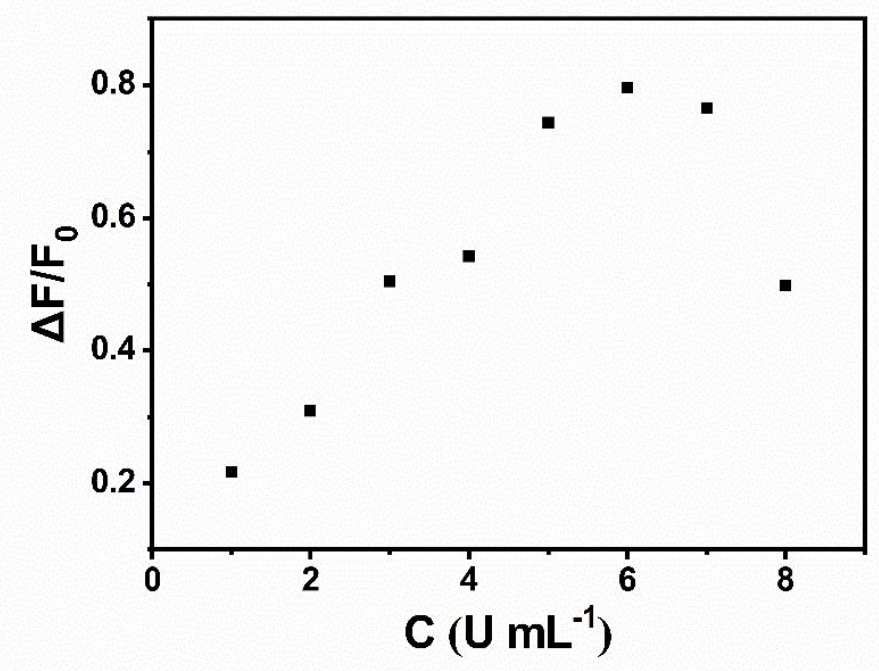

Fig. S8. The effect of concentration of Gal-Ox. Experimental conditions: PF- $b$-PDI, $0.1 \mathrm{mg}$ $\mathrm{mL}^{-1}$; hydroquinone, $20 \mu \mathrm{M}$. 